\title{
SiMULACIÓN DE LA COMPRENSIÓN DE NARRACIONES MEDIANTE LA IMPLEMENTACIÓN DEL MODELO LANDSCAPE Y EL ROL DE LAS INFERENCIAS EMOCIONALES*
}

\section{COMPREHENSION OF NARRATIVES SIMULATION THROUGH THE LANDSCAPE MODEL AND THE ROLE OF EMOTIONAL INFERENCES}

\author{
Juan Pablo Barreyro**, Irene InJoque-Ricle ${ }^{* * *}$ y Carlos Molinari Marotto****
}

\begin{abstract}
*Trabajo realizado en el marco del Proyecto UBACyT 2011-2014 № 20020100200021, otorgado al primer autor. ${ }^{* *}$ Doctor de la Universidad de Buenos Aires (área Psicología). Miembro de la Carrera del Investigador Científico del Consejo Nacional de Investigaciones Científicas y Técnicas (CONICET). Jefe de Trabajos Prácticos de la materia Psicología General en la Facutad de Psicología de la Universidad de Buenos Aires (UBA). E-Mail: jbarreyro@psi.uba.ar Gallo 1569, Piso $8^{\circ}$ Dpto. C - (C1425EFG) Ciudad Autónoma de Buenos Aires. República Argentina.

***Doctora de la Universidad de Buenos Aires (área Salud Mental). Miembro de la Carrera del Investigador Científico del Consejo Nacional de Investigaciones Científicas y Técnicas (CONICET). Ayudante de Trabajos Prácticos de la materia Neurofisiología, en la Facutad de Psicología de la Universidad de Buenos Aires (UBA).

****Doctor de la Universidad de Buenos Aires (área Psicología). Profesor Adjunto de la materia Psicología General en la Facultad de Psicología de la Universidad de Buenos Aires (UBA).

Los autores agradecen a la Dra. Jazmín Cevasco su colaboración en la elaboración de los protocolos de prueba.
\end{abstract}

\section{RESUMEN}

El propósito de este trabajo consistió en estudiar el rol de la generación de inferencias en la comprensión de textos narrativos; para ello se realizaron dos simulaciones con el Programa Landscape sobre un texto narrativo natural, una simulación con arreglo de inferencias emocionales y otra sin ellas, que se pusieron en relación con datos conductuales de sujetos, obtenidos del reconocimiento de las oraciones del texto y de su valoración o relevancia para la historial. Con este propósito, participaron 30 adultos universitarios. Leyeron la narración y posteriormente respondieron a un protocolo de reconocimiento y valoraciones de proposiciones del texto. A continuación se llevó a cabo un análisis de correlaciones y luego un análisis de regresión lineal empleando los valores de proposiciones predichas a partir de dos simulaciones, una simulación causal-referencial (sin emociones) y otra con implementación de inferencias emo- cionales, como variables predictoras, y los valores obtenidos de reconocimiento y valoración y/o relevancia para la historia, como variables dependientes. El análisis de correlación mostró que ambas simulaciones se asociaron con los valores de reconocimiento y valoración, aunque en mayor medida, la simulación con implementación de inferencias emocionales; pero el análisis de regresión detectó que únicamente la simulación con implementación de inferencias emocionales explicó los datos obtenidos de reconocimiento y valoración.

Estos hallazgos sugieren que las inferencias emocionales juegan un rol de importancia en la comprensión de textos narrativos, ya que permiten que el lector focalice su atención hacia determinados puntos de la historia, que serán los nucleares o más importantes.

Palabras clave: Simulación; Adultos; Modelo Landscape; Comprensión de narraciones; Inferencias emocionales. 


\section{ABSTRACT}

Text comprehension requires the construction of a coherent mental representation, integrating text information with previous knowledge. Several studies have suggested that the emotional states of characters need not be stated explicitly: readers can infer them as a consequence of the narrative situation, characters' goals, actions, and relations to other characters. This study investigated emotional inferences generation during reading using the simulation of a narrative text, carried out with the Landscape Program and assessing text sentence recognition and the relevance of the sentence to the story. This program is a connectionist model that represents comprehension as a changing landscape of activations of propositions along several reading cycles. This model proposes that, as the reader procedes through a text, propositions fluctuate in activation. That is, with each reading cycle, new propositions are activated, and activation values of current propositions change. In addition, the coactivation of propositions leads to the establishment of connections between them. Through these fluctuating activations, a memory representation of the text gradually and dynamically emerges. The peaks and valleys of this landscape represent the relative contribution of each proposition at any given point in the story, and are the base for the construction of a mental representation of the story. The aim of this work is to determine if comprehension of a narrative with emotional inferences is a better predictor of textual proposition recognition and its relevance to the story than comprehension of a narrative without emotional inference, using a program that simulates the comprehension process. In order to simulate the generation of emotional inferences, we used the Landscape Computational Model. For this purpose 30 participants, undergraduates (9 males -30\%- and 21 women, with a mean age of 20.67 years, $S D=2.85$ ), read a story and subsequently completed a proposition recognition and proposition relevance for the story protocol. Pearson correlation analysis and linear regression analysis were conducted. Two linear regression models were tested, both including propositional values of simulation with emotional inference and without emotional inferences as independent variables, one including proposition recognition as dependent variable, and the other including proposition relevance for the story as dependent variable. The Pearson correlation coefficients showed that the simulation of the story with emotional interference and the simulation of the story without emotional interference are related to proposition recognition and story relevance, although the relation between this values and the simulation based on emotional inference had better coefficients $(r=.35, p<.01$, and $r=.38, p<.01$ respectively) than the causal-referential simulation ( $r=.27, p<.05$, and $r=.28, p<.05$ respectively). The linear regression analysis detected that only the simulation with emotional inference explained the variance of recognition data $(\beta=.45, p<.01)$ and the variance of the relevance to the story values $(\beta=.53, p<.01)$. These findings suggest that emotional inferences play an important role in the understanding of narratives texts; because they focus the reader's attention to certain important points of the story. That is, the realization of emotional inferences seems to intensify the attention that the reader devotes to the entire cycle (which includes the proposition that prompts the inference, and also those that are causally connected to it), facilitating its later recognition and relevance to the story. This intensified processing can be related to the role that characters' emotional reactions play in a narrative.

Keywords: Simulation; Landscape model; Narrative comprehension; Emotional inferences; Adults.

\section{INTRODUCCIÓN}

La comprensión del texto es una de las más complejas actividades cognitivas humanas y supone la construcción de una representación coherente acerca del contenido textual en una memoria episódica (Kendeou \& van den Broek, 2005, 2007; Kintsch, 1998; Rapp \& van den Broek, 2005; Rapp, van den Broek \& Kendeou, 2005; van den Broek, Virtue, Everson, Tzeng \& Sung, 2002). Para ello, el lector debe conectar e integrar ideas provenientes del texto con su conocimiento previo, esto es generar inferencias 
(van den Broek, 1994). Una amplia gama de investigaciones ha abordado los procesos implicados en la generación de inferencias durante la comprensión de textos, así como la naturaleza de la información que es activada y codificada (Graesser, Singer \& Trabasso, 1994; Singer \& O’Connell, 2003; van den Broek, Rohleder \& Narváez, 1996). Dentro del conjunto de inferencias, las inferencias emocionales que se proponen, juegan un papel importante en la comprensión de las narraciones, dado que implican la activación de conocimientos acerca de los estados emocionales de los personajes de ficción, como consecuencia de los eventos de la historia, que permiten mantener activo el foco de atención del lector hacia los eventos venideros (de Vega, León \& Díaz, 1996; Gernsbacher, 1995; Gygax, Oakhill \& Garnham, 2003; Molinari, Barreyro, Cevasco \& van den Broek, 2011; Molinari et al., 2009).

Un modo de estudiar los procesos involucrados en la comprensión del texto es a través de la realización de simulaciones por medio de programas de computación. Estos programas constituyen descripciones funcionales que especifican las relaciones entre los procesos que operan durante la comprensión, a partir de considerar los resultados de un amplio cuerpo de investigaciones previas. Dentro del conjunto de programas de simulación de la comprensión, el modelo Landscape (van den Broek, Risden, Fletcher \& Thurlow, 1996; van den Broek, Young, Tzeng \& Linderholm, 1999) permite comparar teorías que difieren entre sí en algún aspecto determinado, a partir de contrastar las predicciones hechas por distintos modelos con los datos conductuales. Es decir, permite comparar las respuestas generadas por el programa cuando simula un proceso cognitivo con arreglo a una u otra alternativa teórica de procesamiento con respuestas generadas por sujetos humanos. De este modo, el modelo computacional constituye una plataforma de prueba de alternativas teóricas acerca de algún aspecto de la comprensión.

El propósito del estudio que se informa fue determinar si la simulación de la comprensión de una narración natural con arre- glo de inferencias emocionales es un mejor predictor del desempeño de los participantes en el reconocimiento de proposiciones extraídas del texto y en la valoración de cada proposición en relación al texto, a diferencia de una simulación sin el arreglo de inferencias emocionales.

\section{LAS INFERENCIAS EMOCIONALES}

La generación de inferencias emocionales durante la comprensión de textos no ha recibido la misma atención. Son actualmente, pocos los estudios que han trabajado en la investigación, por ejemplo, de la comprensión en textos narrativos naturales (Barreyro, $\mathrm{Ce}$ vasco, Burin \& Molinari, 2012; Molinari et al., 2011; van den Broek, Rohleder et al., 1996). La gran mayoría se ha centrado en textos experimentales breves creados por el investigador, así resultan mayores las investigaciones experimentales sobre inferencias emocionales en estos textos o pseudotextos (de Vega et al., 1996; Gernsbacher, 1995; Gernsbacher, Goldsmith \& Robertson, 1992; Gernsbacher, Hallada \& Robertson, 1998; Gygax et al., 2003; Molinari et al., 2009).

Uno de los primeros estudios que ha mostrado que los lectores pueden inferir las reacciones emocionales de personajes, como consecuencia de la comprensión de las acciones de estos, sus metas y sus relaciones con otros personajes es el de Gernsbacher y colaboradores (1992). Ellos confeccionaron historias breves en cada una de las cuales un protagonista, por acciones propias u otras circunstancias, se hallaba comprometido en una situación que contenía las condiciones desencadenantes de una emoción. Midieron tiempos de lectura para determinar si la oración final de cada texto, que explicitaba qué emoción sentía el protagonista, era leída más lentamente cuando la emoción mencionada no era la correspondiente a la reacción emocional que el relato implicaba. En tres experimentos examinaron, utilizando el mismo procedimiento, tres diferentes relaciones entre la emoción que se esperaba que el lector infiriese durante la lectura de cada texto 
y la emoción incongruente. En el primer experimento la emoción incongruente era la emoción contraria, por cuanto se le oponía por valencia afectiva, siendo equivalente en otras dimensiones.

En un segundo experimento (Gernsbacher, 1995), la disparidad de ambas emociones se daba no sólo en cuanto a valencia sino también en otras dimensiones, de modo que ya no se trataba de emociones contrarias, sino meramente de emociones de valencia opuesta.

En un tercer experimento, la emoción incongruente era de la misma valencia que la esperable, pero menos probable en la situación descrita. Por ejemplo: esperable: culpa [guilt], contraria: orgullo [pride], valencia opuesta: esperanza [hope], misma valencia: timidez [shyness]. Otro ejemplo: esperable: aburrimiento [boredom], contraria: curiosidad [curiosity], valencia opuesta: audacia [boldness], misma valencia: enojo [anger].

Los resultados de los tres experimentos mostraron la diferencia esperada entre los tiempos de lectura de las oraciones emocionalmente incongruentes y los de las oraciones congruentes. Además, comparando resultados de los tres experimentos, se halló el siguiente patrón: cuanto más alejada era la emoción incongruente respecto de la reacción emocional implicada en cada historia, más lentamente los participantes leían la oración correspondiente. Cuando la emoción incongruente era la emoción contraria, el tiempo de lectura era máximo; cuando ambas emociones eran opuestas en valencia pero no contrarias, los participantes leían la oración incongruente menos lentamente; y cuando eran de la misma valencia afectiva los participantes leían la oración incongruente aún menos lentamente, aunque nunca tan rápido como cuando leían la que explicitaba la emoción esperable, este resultado encontrado es semejante al de otras investigaciones posteriores (Gygax et al., 2003; Molinari et al., 2009).

En otro trabajo, De Vega y colaboradoes (1996) en un primer experimento replicaron los hallazgos de Gernsbacher y colaboradoes (1992) en cuanto a que los lectores infieren la emoción del protagonista de una historia breve, y aportaron evidencia de que la infe- rencia puede actualizarse si cambia la situación que la historia describe. En otros dos experimentos mostraron que, tras haber sido inferida una emoción del protagonista de una historia experimental, la representación de la emoción inferida permanece en primer plano (foregrounded) durante el procesamiento de información subsiguiente sin contenido emocional. De este modo el lector está en condiciones de integrar cualquier información emocionalmente significativa que aparezca posteriormente en el mismo texto, incluso aunque esa nueva información sea una descripción de una conducta emocionalmente motivada sin mención explícita de la emoción. En un tercer estudio, Gernsbacher y colaboradoes (1998) investigaron cuán automáticamente los lectores activaban su conocimiento sobre las emociones durante la lectura de historias breves. Se midieron los tiempos de lectura de oraciones blanco que contenían palabras emocionales congruentes o incongruentes luego que un participante había leído la mitad de la historia mientras realizaba o no una tarea secundaria que consistía en identificar tonos graves y agudos. Los resultados indicaron que las oraciones que contenían una palabra emocional congruente con la historia eran leídas más rápidamente que las oraciones que contenían una palabra emocional incongruente, tanto en los participantes que no realizaban la tarea secundaria, como en aquellos que la realizaban. Esto implica que la capacidad de activar el conocimiento sobre las emociones no parece estar atenuada cuando se lleva a cabo una tarea concurrente, lo que sugiere que los lectores pueden fácilmente acceder a la información emocional sobre los personajes.

\section{EL MODELO LANDSCAPE}

Con el propósito de simular la comprensión del texto con implementación de inferencias emocionales, se utilizó el modelo computacional Landscape (van den Broek et al., 1996; van den Broek et al., 1999). Este modelo tiene una arquitectura computacional conexionista para simular la comprensión, esto es, se vale de unidades cognitivas 
elementales y conexiones entre estas. La activación de las unidades va variando a medida que avanza la lectura y entre ellas se van estableciendo conexiones cuyo peso (la fuerza de cada conexión) también va variando. Al finalizar la lectura, cada una de esas unidades, que en conjunto forman una representación en forma de red, tiene determinada fuerza de nodo, y cada par de unidades tiene determinado peso de conexión, que puede ser cero (conexión nula) o mayor que cero. Esta representación en forma de red no se forma súbitamente al finalizar la lectura, sino que se va construyendo a lo largo de la lectura, hasta llegar a su conformación final. En términos conexionistas, la representación producto de la comprensión ha sido aprendida paso a paso, aprendizaje que culmina en una red de conexiones de pesos diversos entre nodos de fuerza diversa.

Las unidades en el modelo son proposiciones, unidades menores de significado en un texto y a la vez unidades de procesamiento del mismo. Las proposiciones ingresan al modelo en ciclos de lectura, también denominados ciclos de procesamiento. Si se trata de una oración simple, el contenido nuevo del ciclo coincidirá en general con una proposición; si se trata de oraciones compuestas, el contenido nuevo del ciclo consistirá en más de una proposición. En cada nuevo ciclo de lectura hay cuatro fuentes potenciales de activación: (1) la oración que se está procesando, (2) el ciclo de lectura inmediato anterior, (3) proposiciones reactivadas que fueron procesadas en algún ciclo previo y (4) proposiciones activadas desde el conocimiento general del lector.

En cuanto a la primera fuente de activación, las proposiciones contenidas en la oración que se está procesando, es decir, las explícitamente mencionadas en cada ciclo de lectura, son las máximamente activadas. Cada ciclo incorpora por lo menos una nueva proposición. La segunda fuente de activación está dada por las proposiciones del ciclo anterior, que tendrán cierto traspaso de activación al ciclo actual, dado que las activaciones declinan a cero de una manera asintótica. La tercera y cuarta fuentes de activa- ción consisten en proposiciones pertenecientes a ciclos de lectura previos, o bien recuperadas del conocimiento general, que podrán asimismo tener activación en el ciclo actual.

Debido a la capacidad atencional limitada, que hace que la lectura avance por ciclos, y las distintas fuentes de activación mencionadas, la activación de las proposiciones fluctúa constantemente a medida que avanza la lectura. En cada nuevo ciclo hay activación de nuevas proposiciones, otras mantienen o recuperan activación y otras son desplazadas del foco de atención o de la memoria de trabajo. Considerando simultáneamente los picos y valles de activación de cada proposición a lo largo de los ciclos de lectura, se obtiene un paisaje de activaciones, que da nombre al modelo.

Una característica distintiva del modelo Landscape es la activación de cohorte, cuando una proposición es activada, otras que están conectadas con ella recibirán cierta activación también. El monto de activación de cada una de las proposiciones secundariamente activadas es una función de la fuerza de su relación con la proposición primariamente activada y del monto de activación actual de esta última. Además, un parámetro de activación de cohorte modula el alcance de la transferencia de activación de una proposición a su cohorte (van den Broek et al., 1999; van den Broek et al., 2002). La cohorte de una proposición en un punto determinado del texto difiere de su cohorte en otro momento, así como difiere también la activación transmitida a la cohorte. De este modo, así como las activaciones de cada nuevo ciclo van determinando la emergencia de la representación, la representación emergente con sus conexiones ya establecidas hasta el momento va a su vez modulando las activaciones de cada nuevo ciclo.

Se pueden distinguir tres fases de la simulación de la comprensión mediante el modelo Landscape. La primera corresponde a la determinación de los valores de input para cada proposición, sobre la base del análisis de la estructura textual. En la segunda el modelo procesa ciclo por ciclo esos valores 
de input y produce vectores de activación que incluyen la activación de cohorte. En la tercera el modelo, también ciclo por ciclo, va estableciendo y actualizando las conexiones de la emergente red interconectada de memoria. Las fases segunda y tercera interactúan entre sí a medida que se van computando los valores de input iniciales. El output del modelo son dos matrices, una de las cuales contiene los valores de activación on-line (que constituyen el paisaje de activaciones propiamente dicho) y la otra las conexiones off-line entre nodos que constituyen la representación de memoria episódica aprendida durante la lectura, es decir, el resultado final de la comprensión.

Investigaciones previas han aplicado el modelo Landscape al estudio de la comprensión textos, van den Broek, Risden y colaboradores (1996) compararon las predicciones que realiza el modelo, contemplando la generación de inferencias causales y referenciales (van den Broek, Risden, \& Husebye-Hartmann, 1995) con las de activación de conceptos online durante la lectura y con los puntajes de activación provistos por un grupo de lectores. Esto es, se solicitó a un grupo de participantes que leyeran una narración y estimaran cuán activo pensaban que se encontraba cada concepto luego de la lectura de cada oración. Se encontró una correlación alta entre las predicciones del modelo y las estimaciones de los sujetos. Los conceptos que eran más recordados fueron aquellos que tenían una mayor activación en memoria en la predicción hecha por la simulación. En cuanto a la comprensión de textos expositivos, se encontró a su vez que las proposiciones que tenían los valores más altos en la representación simulada de la comprensión eran las más recordadas (van den Broek et al., 2002). La detección de inconsistencias o contradicciones en el texto también fue estudiada a través del Landscape (Linderholm, Virtue, Tzeng \& van den Broek, 2004; van den Broek et al., 1999) y se observó que la probabilidad de que un lector detecte la contradicción entre la información dada inicialmente y la información dada posteriormente dependía de la activa- ción que hubiera recibido la información inicial, la fuerza de sus conexiones con otros conceptos del texto y la superposición semántica entre la información inicial y la información posterior. Los propósitos de lectura (estudio - entretenimiento) también fueron examinados a través de la realización de simulaciones alternativas (Linderholm et al., 2004). Se observó que la simulación que contemplaba el propósito del estudio resultaba en una mayor activación general de los conceptos que formaban parte del texto que la simulación que contemplaba el propósito de entretenimiento. En especial, las ideas principales del texto recibieron más activación en la simulación de estudio. La comparación de estas simulaciones con los datos conductuales mostró que las predicciones del modelo capturaban correctamente los procesos inferenciales de los lectores en función de sus propósitos para leer el texto.

\section{MÉTOdO \\ PARTICIPANTES}

Se trabajó con 30 alumnos de primer año de la Universidad de Buenos Aires (9 varones y 21 mujeres), que participaron voluntaria y anónimamente de la experiencia a cambio de un crédito académico durante el transcurso de la materia Psicología General. El promedio de edad de la muestra fue igual a 20.67 años $(D E=2.85)$. La muestra fue intencional y los participantes dieron su consentimiento para participar en la investigación.

\section{Materiales}

El material narrativo consistió en la traducido al español de un cuento popular alemán, de la colección Kinder- und Hausmärchen compilada por Jacob y Wilhelm Grimm (1812): "Juliana y Julián" (en el original Jorinde y Joringel). El cuento contenía una extensión de 38 oraciones y 74 proposiciones. En base al cuento se confeccionó una prueba de reconocimiento de proposiciones del 
texto, en la que el participante debía indicar el nivel de importancia o relevancia (valoración) de esa proposición para la historia en una escala de 1 a 5 , siendo 1 muy poco relevante para la historia y 5 muy relevante.

En el Anexo 1 se presenta un fragmento de la historia y en el Anexo 2, una parte de la prueba de reconocimiento y valoración de la relevancia para la historia de cada proposición.

\section{PROCEDIMIENTO DE SIMULACIÓN}

El modelo Landscape, como ya se mencionó, sirve como plataforma de prueba de alternativas teóricas acerca de la comprensión. En este estudio se realizaron dos simulaciones alternativas de la comprensión del cuento, utilizando el Programa Landscape 1.3 para Windows (van den Broek et al., 1999; van den Broek et al., 2002). La diferencia entre ambas simulaciones fue la implementación de inferencias emocionales en una de ellas. Más información acerca de la construcción de la simulación puede encontrarse en el trabajo de Molinari y colaboradores (2011). Estudios previos (Tzeng, 2007; van den Broek et al., 1996; van den Broek et al., 1999; van den Broek et al., 2002) sugieren que la implementación de una teoría de generación de inferencias causal - referencia (van den Broek, 1994; van den Broek, Rohleder \& Narváez, 1996), que supone que el lector utiliza sistemáticamente los estándares de coherencia de claridad anafórica y explicación suficiente, es la mejor predictora de los resultados de medidas conductuales online y off-line. Esta simulación es la que se utilizó como base para la investigación a comparar. Se llamará simulación causal-referencial a la simulación que no implementa inferencias emocionales. Para esta simulación los valores de input fueron los siguientes: un valor 5 para las nuevas proposiciones mencionadas en cada ciclo, un valor de 4 para la reactivación de proposiciones previas correferenciales con alguna nueva proposición y para la reactivación de proposiciones previas que tienen un vínculo causal fuerte con el contenido del ciclo actual, un valor de 3 para la reactivación de proposiciones previas que tienen un vínculo causal débil o de posibilitación, esto es, el tipo de relación causal entre las dos proposiciones es necesario pero no suficiente en las condiciones de la historia para generar la relación causal. La simulación que implementa las inferencias emocionales no constituye un cambio radical con respecto a la que no las implementa, sino que agrega una categoría de inferencias. Para la implementación de las inferencias emocionales se ha considerado resaltar en activación aquellas oraciones que contienen una inferencia emocional, otorgándole una mayor importancia, asumiendo que esta categoría de inferencias tiene una función distinta, ya que en términos de Gernsbacher (1995), una inferencia emocional es un elemento acerca de estados emocionales concomitantes que intensifican la atención del lector en ese ciclo. Para la simulación con implementación de inferencias emocionales, se basó en la simulación causal - referencial más emoción. Para esta simulación, por lo tanto, los valores de input son diferentes según se trate de un ciclo de lectura emocional o no. Los ciclos de lectura no emocionales mantienen los valores de input de la simulación antes descrita. Los ciclos de lectura emocionales tienen los siguientes valores de input: un valor de 6 para las nuevas proposiciones mencionadas en el ciclo, un valor de 5 para reactivación de proposiciones previas correferenciales con alguna nueva proposición y para la reactivación de proposiciones previas que tienen un vínculo causal fuerte, un valor de 4 para la reactivación de proposiciones previas que tienen un vínculo causal débil con el ciclo actual.

\section{Procedimiento}

En una única sesión individual los participantes leían la historia a su propio ritmo y a continuación completaban la prueba de reconocimiento y valoración de la relevancia de las proposiciones del texto. 


\section{ANÁLISIS DE DATOS}

Todos los análisis se realizaron tomando como unidad a cada una de las 74 proposiciones extraídas de la narración. Para cada una de ellas se obtuvo su valor de activación en memoria episódica que se obtiene del valor de conexiones off-line, del resultado final de la simulación, tanto para la simulación causal-referencial, como para la simulación con implementación de inferencias emocionales.

También se calculó su proporción de reconocimiento correcto a partir de la prueba de reconocimiento, y su promedio de valoración de la relevancia para la historia obtenido, también, de las pruebas realizadas por los participantes. A partir de estos puntajes, valor de activación en memoria de una simulación causal-referencial, de una simulación con implementación de inferencias emocionales, el porcentaje de reconocimiento correcto y el promedio de relevancia para la historia, se calcularon los estadísticos descriptivos, se analizaron las distribuciones y se llevaron a cabo análisis de correlación y de regresión lineal múltiple con el propósito de conocer cuál de las dos simulaciones se relaciona en mayor medida con las puntuaciones de reconocimiento y valoración de la relevancia para la historia, y en segundo lugar cuál de las dos simulaciones explica mejor estos resultados.

Para los análisis de regresión lineal múltiple, se tomaron como variables predictoras los valores de activación de las proposiciones en memoria para una simulación causal referencial y para una simulación con implementación de inferencias emocionales, $y$ como variables predichas los valores obtenidos de la prueba de reconocimiento y de relevancia para la historia.

\section{Resultados}

En primer lugar, se analizaron las distribuciones de los puntajes y se obtuvieron los estadísticos descriptivos de cada medida obtenida de las simulaciones y de las pruebas de reconocimiento y valoración de la rele- vancia para la historia. Respecto del análisis de las distribuciones, se observó que los valores de activación en memoria para las proposiciones en una simulación causal-referencial se alejaron significativamente de los valores de una distribución normal asintótica $\left(K-S_{(75)}=2.09 ; p<.001\right)$. Por esta razón para los análisis siguientes se procedió a su normalización a partir de su transformación al logaritmo natural. En la Tabla 1 se informan los estadísticos descriptivos de los valores analizados de las proposiciones y de normalidad mediante la prueba $Z$ de Kolmogov- Smirnov $(K-S)$.

Con el propósito de conocer cuál de las dos simulaciones realizadas, con implementación de inferencias emocionales o sin ellas, se relaciona con las puntuaciones obtenidas se llevó a cabo un análisis de correlaciones, empleando el estadístico $r$ de Pearson. A partir de este análisis se observa que los resultados de ambas simulaciones se asocian con las puntuaciones de reconocimiento y de relevancia para la historia. Pero los valores de activación de cada proposición en la representación mental predichos por la simulación causal-referencial presentan asociaciones de baja intensidad y menores con reconocimiento y relevancia, en comparación con los valores obtenidos de la correlación a partir de los predichos por la simulación con implementación de inferencias emocionales. En la Tabla 2 se presenta el resultado del análisis de correlación entre las medidas obtenidas de cada una de las simulaciones y los puntajes de reconocimiento y relevancia.

Al observar los datos obtenidos con el análisis de correlaciones, y con el propósito de conocer cuál de las dos predicciones hechas a partir del programa de simulación explica mejor los puntajes obtenidos de reconocimiento y valoración de la relevancia para la historia, se realizaron dos análisis de regresiones. Para estos análisis se tomaron como variables predictoras los valores de activación de las proposiciones en memoria para una simulación causal referencial y para una simulación con implementación de inferencias emocionales, y como varia- 
bles predichas los valores obtenidos de la prueba de reconocimiento y de relevancia para la historia.

El análisis de regresión lineal sobre los puntajes obtenidos de reconocimiento muestra en primer lugar, que el modelo fue estadísticamente significativo $\left[F_{(2,72)}=5.16\right.$, $M S E=0.99, p<.01, R=.35$ ], en segundo lugar, que la simulación causal-referencial no explicó significativamente los resultados de la prueba de reconocimiento, mientras sí lo hizo la simulación con implementación de inferencias emocionales. El análisis de regresión sobre los puntajes obtenidos de la prueba de valoración de la relevancia para la historia de cada proposición muestra que el modelo fue también, estadísticamente significativo $\left[F_{(2,72)}=6.39, M S E=31.24, p<\right.$ $.01, R=.39]$, y al igual que el análisis anterior, la simulación causal-referencial no explicó los resultados de las variancias de la prueba de valoración, como sí lo muestran los resultados de la simulación con implementación de inferencias emocionales.

En la Tabla 3 pueden observarse los resultados de ambos modelos de regresión realizados.

\section{Discusión}

El modelo Landscape (Tzeng, 2007; Tzeng, van den Broek \& Kendeou, 2005; van den Broek et al., 1999; van den Broek et al., 2002) por su arquitectura conexionista, concibe a la representación mental del texto como una red de proposiciones unidas por relaciones de fuerzas de nodo, interconectadas con pesos de conexión. Esa red constituye el resultado de un gradual aprendizaje a lo largo de varios ciclos de lectura durante los cuales las proposiciones han sido activadas repetidamente y en distinta medida. Por decirlo así, la red aprende que en la historia, algunas proposiciones son más importantes que otras, esto es, más centrales, nucleares. También aprende que los posibles pares de proposiciones se vinculan entre sí con diferente peso de conexión. Si se concibe a la representación mental de la historia como una red así conformada, esa red es la que permite a un participante de una prueba de recuerdo libre reconstruir la historia original de una manera proposicional y situacionalmente adecuada.

Cuando se realiza una simulación en el modelo Landscape, se obtiene una matriz de activación, que contiene los vectores de activación correspondientes a cada ciclo de lectura y una matriz de conexiones que contiene las fuerzas de nodo y pesos de interconexiones entre todos los posibles pares de proposiciones, esta matriz es la que representa la fuerza de cada proposición en la memoria episódica del lector (Molinari et al., 2011; Tzeng, 2007). Si algún factor tiene influencia sobre los valores definitivos de esas matrices, su manipulación permitirá obtener distintos valores predictores de datos conductuales on-line y off-line. El mayor ajuste de una de las simulaciones con efectivos datos conductuales aporta evidencia a favor del papel funcional de determinado factor durante la comprensión de determinada clase de textos.

Una manipulación de estas características fue la que se realizó en este trabajo, donde dos simulaciones alternativas de la comprensión de narraciones, una simulación causal-referencial y otra además con implementación de inferencias emocionales, se emplearon para estudiar la relación con protocolos de reconocimiento de proposiciones de una historia y de valoración de la relevancia para la historial. Hay que recordar que las dos simulaciones difirieron en su implementación, en una de ellas de inferencias emocionales, mediante el recurso de aumentar sistemáticamente los valores de input de las distintas fuentes de activación sólo en los ciclos emocionales, manteniendo en los demás ciclos los mismos valores de input. Esta fue una manera de simular la función de incremento de recursos atencionales, o intensificación de la atención global, durante esos ciclos, por el solo hecho de que son ciclos en los que se infiere una emoción de un personaje.

Los resultados de las correlaciones, mostraron un buen ajuste de ambas simulaciones 
con los datos provenientes del reconocimiento y de la valoración de la relevancia de la proposición para la historia. Este resultado es congruente con la investigación realizada sobre el modelo Landscape (Linderholm et al., 2004; Molinari et al., 2011; Tzeng, 2007; Tzeng et al., 2005; van den Broek, Risden et al., 1996; van den Broek et al., 1999). Pero las correlaciones también mostraron una mejor asociación de los datos con la simulación con implementación de inferencias emocionales, resultado coherente con investigaciones realizadas a partir de la evaluación de los recuerdos de historias (Molinari et al., 2011), en donde se ha obsevado que el resultado de un modelo de simulación con arreglo de inferencias emocionales está más asociado con el recuerdo de oraciones, que un modelo de simulación sin arreglo de inferencias emocionales.

Por otro lado, el análisis de regresión mostró que únicamente los valores predichos de comprensión realizados por la simulación con implementación de inferencias emocionales pudieron explicar las variancias obtenidas de los datos de reconocimiento y valoración y/o relevancia.

Stein y Trabasso (1992) afirman que las emociones están relacionadas con el estado de las metas valoradas y los acontecimientos que pueden afectar ese estado. Desde este punto de vista, las emociones de personajes son inferidas atendiendo a la estructura causal de la historia, lo cual supone un monitoreo del estado de las metas, intentos (acciones) y resultados de esos intentos, que dan forma a los episodios, los cuales disparan valoraciones del lector acerca de la situación. Las inferencias emocionales se generan por asociaciones basadas en conocimientos acerca de la potencial capacidad de las situaciones para provocar una emoción. Pero una vez inferida la emoción, no queda sólo como un elemento más que se incluye en la representación de la historia. La inferencia emocional ejerce influencia sobre el vector de activaciones del ciclo de lectura durante el cual se generó. El vector completo del ciclo emocional recibirá un plus de activación, con las consecuencias que ello implica para la representación final. La lógica del modelo hace que algunas fuerzas de nodo que en la representación final resultan realzadas por este plus de activación de los ciclos emocionales no sean evidentes a primera vista. De hecho, algo que la simulación pudo reflejar adecuadamente es la hipótesis de que la inferencia emocional acrecienta los recursos atencionales aplicados al ciclo, esto es, no a determinadas proposiciones en cuanto tales, sino al vector de activación del ciclo emocional. Así algunas proposiciones recibieron el plus de activación en algunos ciclos y no en otros.

Esta ventaja de activación de algunos ciclos de lectura les da mayor realce, lo que permite que se reconozcan en una mayor proporción, y además que el sujeto las identifique como más relevantes para la historia. En definitiva, la ventaja empírica de una simulación por comparación con la otra brinda un apoyo a la hipótesis de la función de incremento atencional de las inferencias de emociones de personajes.

Si bien la investigación realizada fue llevada a cabo con una muestra de jóvenes adultos universitarios que eran hablantes nativos del español, investigaciones posteriores podrían estudiar e intentar testear modelos de procesamiento cognitivo en simulación teniendo en cuenta diferencias de conocimiento previo, nivel socioeconómico, y ver qué ocurre durante el desarrollo cognitivo en niños, asimismo testear la hipótesis de incremento atencional empleando otras medidas conductuales. 
TABLA 1

ESTADÍSTICOS DESCRIPTIVOS DE LOS VALORES DE PROPOSICIONES

\begin{tabular}{|l|ccccc|}
\hline & $M$ & $D E$ & $A$ & $C$ & $K-S$ \\
\hline Simulación causal - referencial & 1.32 & .37 & .96 & .37 & $2.09^{* * *}$ \\
Simulación con implementación & & & & .69 & 1.17 \\
de inferencias emocionales & 1.09 & .34 &. .78 & -.12 & 1.24 \\
Reconocimiento & .85 & .12 & -.84 & .18 & .94 \\
Relevancia para la historia & 3.54 & .71 & & & \\
\hline
\end{tabular}

${ }^{* * *} p<.001$

TABLA 2

CORRELACIONES ENTRE LAS MEDIDAS DE SIMULACIÓN Y RECONOCIMIENTO Y VALORACIÓN

\begin{tabular}{|l|cc|}
\hline & Reconocimiento & Relevancia para la historia \\
\hline Simulación causal - referencial & $.27^{*}$ & $.28^{*}$ \\
Simulación con implementación de inferencias emocionales & $.35^{\star *}$ & $.38^{* *}$ \\
\end{tabular}

${ }^{* *} p<.01$

${ }^{*} p<.05$

TABLA 3

RESULTADOS DEL ANÁLISIS DE REGRESIÓN LINEAL

\begin{tabular}{|l|c|c|c|}
\hline & $\begin{array}{c}\text { Simulación } \\
\text { causal - referencial } \\
\beta\end{array}$ & $\begin{array}{c}\text { Simulación con } \\
\text { inferencias emocionales } \\
\beta\end{array}$ & $R$ \\
\hline Reconocimiento & -.11 & $.45^{\star *}$ & .35 \\
Relevancia para la historia & -.17 & $.53^{\star *}$ & .39 \\
\hline
\end{tabular}

${ }^{* *} p<.01$ 


\author{
ANEXO1 \\ FRAGMENTO DEL TEXTO “JULIANA Y JULIÁN"
}

"Érase una vez una bruja que vivía en un viejo palacio en medio de un bosque muy espeso. De día se convertía en búho y por la noche recuperaba su aspecto humano. A quien se acercaba a cien pasos del palacio lo inmovilizaba. Si una doncella se acercaba, la bruja la transformaba en pájaro, la encerraba en una cesta y la llevaba al palacio. Tenía más de mil cestas de esta clase.

Un día dos enamorados, Juliana y Julián, fueron a pasear al bosque. Era una bella tarde, el sol brillaba entre las ramas de los árboles y los pájaros cantaban desde lo alto. Caminaron hasta que el sol comenzó a ocultarse. De pronto Julián vio que se acercaban al viejo muro del palacio. Miró hacia donde estaba Juliana, pero ella se había convertido en un ruiseñor que cantaba. Un búho voló tres veces a su alrededor. Julián quedó allí paralizado, sin poder hablar, ni mover las manos ni los pies...".

\title{
ANEXO 2
}

\section{Fragmento del protocolo de la Prueba de Reconocimiento y Valoración de La oración}

Género:

Edad:

Consigna: El objetivo de este cuestionario es identificar qué oraciones formaban parte del texto leído y cuáles no, marcando "si", si consideras que la oración era parte del texto, y "no" si no formaba parte del mismo. De aquellas que has considerado que formaron parte del texto, te pedimos que indiques cuán importante o centrales consideras que eran en la historia, marcando desde 1, si piensas que es poco importante hasta 5 si piensas que es muy importante.

\begin{tabular}{l|c|c|}
\hline \multicolumn{1}{c|}{ Juliana y Julián } & & $\begin{array}{c}\text { Nivel de importancia } \\
\text { de la oración }\end{array}$ \\
\hline \multicolumn{1}{c|}{ Cláusula } & Sí / No & 1 \\
\hline & & \\
\hline 1.- Érase una vez una bruja que vivía en un viejo palacio en & & \\
medio de un bosque muy espeso. \\
2.- Había una vez una vieja bruja que deseaba la juventud. \\
3.- De día se convertía en búho \\
4.- y por la noche recuperaba su aspecto humano. \\
5.- Con sus trapos paseaba por el palacio. \\
6.- A quien se acercaba a cien pasos del palacio \\
7.- le pedía que se quedara quieto. \\
8.- Lo inmovilizaba. \\
9.- Y lo ataba.
\end{tabular}




\section{REFERENCIAS BIBLIOGRÁFICAS}

Barreyro, J.P., Cevasco, J., Burin, D. \& Molinari, C. (2012). Working memory capacity and individual differences in the making of reinstatement and elaborative inferences. The Span ish Journal of Psychology, 15(2), 471-479. http://dx.doi.org/10.5209/rev_SJOP.2012.v15.n 2.38857

de Vega, M., León, I. \& Díaz, J.M. (1996). The representation of changing emotions in reading comprehension. Cognition and Emotion, 10, 303-321. http://dx.doi.org/10.1080/02699939 6380268

Gernsbacher, M.A. (1995). Activating knowledge of fictional characters' emotional states. En C.A. Weaver, S. Mannes \& C.R. Fletcher (Eds.), Discourse comprehension. Essays in honor of Walter Kintsch (pp. 141-156). Hillsdale, NJ: Lawrence Erlbaum.

Gernsbacher, M.A., Goldsmith, H.H. \& Robertson, R.R. (1992). Do readers mentally represent characters' emotional states? Cognition and Emotion, 6, 89-111.

Gernsbacher, M.A., Hallada, B.M. \& Robertson, R.R. (1998). How automatically do readers infer fictional characters' emotional states? Scientific Study of Reading, 2, 271-300. http:// dx.doi.org/10.1207/s1532799xssr0203_5

Graesser, A.C., Singer, M. \& Trabasso, T. (1994). Constructing inferences during narrative text comprehension. Psychological Review, 101, 371-395. http://dx.doi.org/10.1037/0033-295 X.101.3.371

Gygax, P., Oakhill, J. \& Garnham, A. (2003). The representation of characters' emotional responses: Do readers infer specific emotions? Cognition and Emotion, 17, 413-428. http:// dx.doi. rg/10.1080/02699930244000048

Kendeou, P. \& van den Broek, P. (2005). The effects of readers' misconceptions on comprehension of scientific text. Journal of Educational Psychology, 97, 235-245. http://dx.doi. org/10.1037/0022-0663.97.2.235

Kendeou, P. \& van den Broek, P. (2007). The effects of prior knowledge and text structure on comprehension processes during reading of scientific texts. Memory \& Cognition, 35(7), 1567-1577.

Kintsch, W. (1998). Comprehension. A paradigm for cognition. Cambridge: Cambridge University Press.

Linderholm, T., Virtue, S., Tzeng, Y. \& van den Broek, P. (2004). Fluctuations in the availability of information during reading: Capturing cognitive processes using the Landscape Model. Discourse Processes, 37, 165-186. http://dx.doi.org/10.1207/s15326950dp3702_5

Molinari, C., Barreyro, J.P., Cevasco, J. \& van den Broek, P. (2011). Generation of emotional inferences during text comprehension: Behavioral data and implementation through the Landscape model. Escritos de Psicología, 4(1), 9-17. http://dx.doi.org/10.5231/psy.writ.2011. 1803

Molinari, C., Burin, D., Saux, G., Barreyro, J.P., Irrazábal, N., Bechis, M.S. et al. (2009). Fictional characters' emotional states representation: What is its degree of specificity? Psicothema, 21(1), 9-14.

Rapp, D.N. \& van den Broek, P. (2005). Dynamic text comprehension. Current Directions in Psychological Science, 14, 276-279. http://dx. doi.org/10.1111/j.0963-7214.2005.00380.x

Rapp, D.N., van den Broek, P., \& Kendeou, P. (2005). Integrating memory-based and constructionist processes in accounts of reading comprehension. Discourse Processes, 39, 299316.

Singer, M. \& O'Connell, G. (2003). Robust inference processes in expository text comprehension. European Journal of Cognitive Psychology, 15(4), 607-631. http://dx.doi.org/10. 1080/095414400340000079

Stein, N.L. \& Trabasso, T. (1992). The organization of emotional experience: Creating links among emotion, thinking, language, and intentional action. Cognition and Emotion, 6, 225-244. http://dx.doi.org/10.1080/02699939208411070

Tzeng, Y. (2007). Memory of narrative texts: How parts of Landscape model work. Chinese Journal of Psychology, 49, 1-25. 
Tzeng, Y., van den Broek, P. \& Kendeou, P. (2005). The computational implementation of the Landscape model: Modeling inferential processes and memory representations of text comprehension. Behavior Research Methods, 37(2), 277-286. http://dx.doi.org/10.3758/BF0 3192695

van den Broek, P. (1994). Comprehension and memory of narrative texts: Inferences and coherence. En M.A. Gernsbacher (Ed.), Handbook of psycholinguistics (pp. 539-588). San Diego: Academic Press.

van den Broek, P., Risden, K.C., Fletcher, C.R. \& Thurlow, R. (1996). A "Landscape" view of reading: Fluctuating patterns of activation and the construction of a stable memory representation. En B.K. Britton \& A.C. Graesser (Eds.), Models of understanding text (pp. 165-187). Mahwah, NJ: Lawrence Erlbaum.

van den Broek, P., Risden, K.C. \& HusebyeHartmann, E. (1995). The role of readers' standards for coherence in the generation of inferences during reading. En R.F. Lorch \& E.J. O'Brien (Eds.), Sources of coherence in reading (pp. 353-373). Hillsdale, NJ: Lawrence Erlbaum.

van den Broek, P., Rohleder, L. \& Narváez, D. (1996). Causal inferences in the comprehension of literary text. En R.J. Kreuz \& M.S. Mac Nealy (Eds.), Empirical approaches to literature and aesthetics (pp. 179-200). Norwood, New Jersey: Ablex Publishing Corporation.

van den Broek, P., Virtue, S., Everson, M., Tzeng, Y. \& Sung, Y.C. (2002). Comprehension and memory of science texts: Inferential processes and the construction of a mental representation. En J. Otero, J.A. Leon \& A.C. Graesser (Eds.), The psychology of science text comprehension (pp. 131-154). Mahwah, NJ: Erlbaum.

van den Broek, P., Young, M., Tzeng, Y. \& Linderholm, T. (1999). The Landscape model of reading: Inferences and the online construction of memory representation. En H. van Oostendorp \& S.R. Goldman (Eds.), The construction of mental representations during reading (pp. 71-98). Mahwah, NJ: Lawrence Erlbaum.

Universidad de Buenos Aires (UBA)

Consejo Nacional de Investigaciones

Científicas y Técnicas (CONICET)

Ciudad Autónoma de Buenos Aires - República Argentina

Fecha de recepción: 11 de septiembre de 2013

Fecha de aceptación: 11 de febrero de 2014 